\title{
The association between thyroid disorders and incident gout: population-based case-control study
}

\author{
Saskia G Bruderer ${ }^{1,2}$ \\ Christoph R Meier ${ }^{1-3}$ \\ Susan S Jick ${ }^{3}$ \\ Michael Bodmer ${ }^{1,4}$ \\ 'Basel Pharmacoepidemiology \\ Unit, Division of Clinical Pharmacy \\ and Epidemiology, Department of \\ Pharmaceutical Sciences, University \\ of Basel, ${ }^{2}$ Hospital Pharmacy, \\ University Hospital Basel, Basel, \\ Switzerland; ${ }^{3}$ Boston Collaborative \\ Drug Surveillance Program, Boston \\ University School of Public Health, \\ Lexington, MA, USA; ${ }^{4}$ Division \\ of General Internal Medicine, \\ Zug Community Hospital, Baar, \\ Switzerland
}

This article was published in the following Dove Press journal:

Clinical Epidemiology

6 April 2017

Number of times this article has been viewed

Context: Thyroid hormones influence kidney function and thereby might alter serum urate levels, a major risk factor for gouty arthritis.

Objective: To assess the risk of developing incident gout in association with hypothyroidism or hyperthyroidism.

Design: Retrospective population-based case-control analysis..

Setting: UK-based Clinical Practice Research Datalink, a primary care research database.

Patients: We identified adult patients with a diagnosis of incident gout between 1990 and 2014. We matched one control to each gout case in terms of age, sex, general practice, calendar time, and years of active history in the database.

Main outcome measure: We used conditional logistic regression to calculate odds ratios (ORs) with 95\% confidence intervals (CIs) for developing gout in association with hypo- or hyperthyroidism and adjusted for potential confounders.

Results: The study population encompassed 68,159 incident gout cases, of whom $78.8 \%$ were male, and the same number of matched controls. There was no increased risk of gout in patients with hypothyroidism: adjusted OR of gout of 1.12 (95\% CI 1.05-1.20) compared with no hypothyroidism. Current short-term treatment of thyroid hormone replacement therapy was associated with an adjusted OR of gout of 1.54 (95\% CI 1.24-1.92), compared with no treatment. Neither hyperthyroidism nor current treatment with thyroid suppression therapy was associated with gout (adjusted OR, 1.08 [95\% CI 0.95-1.22] and 0.82 [95\% CI 0.57-1.17], respectively). Conclusion: This large observational study does not provide evidence that hypothyroidism or hyperthyroidism, irrespective of treatment, is associated with a clinically relevant increased risk of developing incident gout. There may be an exception among patients with newly diagnosed and treated hypothyroidism.

Keywords: hypothyroidism, hyperthyroidism, Clinical Practice Research Datalink, epidemiology

\section{Introduction}

Gout is a painful rheumatic disease characterized by deposition of uric acid crystals in the joints and elevation of serum uric acid levels, leading to acute inflammatory arthritis. ${ }^{1,2}$ The estimated prevalence of gout in the UK population is $1.4 \%,{ }^{3,4}$ with increasing prevalence in older age groups and with a male-to-female ratio of 3.6-1.3 Various reasons for the difference between males and females in the etiology of the disease have been postulated. ${ }^{5,6}$

Several case series provided evidence of a possible association between hypothyroidism and gout. ${ }^{7-9}$ One study reported a material association between hypo- and hyperthyroidism and the risk of gout, ${ }^{10}$ and two other studies reported a correlation
Correspondence: Christoph R Meier Basel Pharmacoepidemiology Unit, University Hospital Basel, Spitalstrasse 26, $\mathrm{CH}-403$ I Basel, Switzerland

Tel +4I 6I 5565369

$\mathrm{Fax}+41612658875$

Email christoph.meier@usb.ch 
between thyroid disorders and hyperuricemia, an important risk factor for the development of gout. ${ }^{11,12}$ The association between hypothyroidism and hyperuricemia has been firmly established, whereas the relationship between hyperthyroidism and hyperuricemia remains controversial. ${ }^{11}$ A possible explanation is that thyroid hormones influence serum urate levels by regulation of glomerular filtration rate (GFR). ${ }^{13}$ The effects of thyroid hormones on kidney function are the result of direct renal effects, as well as of metabolic and cardiovascular effects. ${ }^{13}$ Hypothyroidism decreases and hyperthyroidism increases GFR, resulting in increased serum urate levels in patients with hypothyroidism and in decreased serum urate levels in patients with hyperthyroidism. ${ }^{13}$ However, a previous small study showed that hyperthyroidism can cause a significant increase in serum uric acid, although less so than hypothyroidism. ${ }^{11}$ Furthermore, one study reported a weak association between thyroid stimulating hormone (TSH) and serum uric acid levels. ${ }^{14}$

Although the association between hypo- and hyperthyroidism and kidney function has been described in the literature, the risk of clinically manifest gout in association with hypo- or hyperthyroidism has not been comprehensively examined. Additionally, the association between use of thyroid hormone replacement or suppression therapy and gout has not yet been studied. We therefore assessed the risk of developing incident gout in association with hypo- or hyperthyroidism and thyroid hormone replacement or suppression therapy, respectively.

\section{Patients and methods}

\section{Data source}

We derived data from the UK-based Clinical Practice Research Datalink (CPRD), a large primary care database that was established in 1987, encompassing data from $\sim 600$ general practices and 10 million patient records. Enrolled patients are representative of the UK population regarding age, sex, and ethnicity. ${ }^{13}$ The CPRD contains information on patient demographics and characteristics, lifestyle variables, such as medical diagnoses, prescriptions, body mass index (BMI), smoking status, and alcohol consumption, symptoms, referrals to consultants, and hospitalizations. The general practitioners generate drug prescriptions directly from the computer, using a coded drug dictionary. ${ }^{15}$ The CPRD has been described in detail elsewhere ${ }^{16,17}$ and has been validated extensively. ${ }^{18-22}$ It has been the source of numerous epidemiological studies published in peer-reviewed journals, including research on gout. ${ }^{3,23-28}$

This study has been approved by the Independent Scientific Advisory Committee for the Medicines and Healthcare
Products Regulatory Agency database research (protocol number 15_079R). The committee did not require written informed consent be obtained from the participants, as this was a retrospective study, and all data was anonymized.

\section{Study population}

\section{Case patients}

Based on Read codes, we identified patients in the CPRD aged $\geq 18$ years who had a first-time diagnosis of gout recorded between 1990 and 2014. The date of the first diagnosis of gout was referred to as the "index date". We excluded all cases with $<3$ years of recorded history prior to the index date to reduce the likelihood of including prevalent gout cases. We further excluded all patients with a diagnosis of HIV or cancer (except nonmelanoma skin cancer) prior to the index date, as well as those with a differential diagnosis of gout such as hemochromatosis, septic arthritis, rheumatoid arthritis, or osteoarthritis at any time within their patient record. We did this to reduce the risk of misclassification, i.e., including alleged gout patients who in fact had another disease with similar symptoms. The diagnosis of gout in the CPRD has been validated in a previous study by review of medical records of incident gout. ${ }^{27}$ They determined gout patients with an incident diagnosis of gout without elevated urate level or treatment against elevated urate levels as we used it as probable cases. ${ }^{27}$ However, the comparison of probable and confirmed cases resulted in similar results. ${ }^{27}$ Similar case definitions to ours have been used by several authors in previous studies. . $^{3,23-26,28}$

\section{Control patients}

We identified at random from the CPRD a control group of patients without any Read code for gout at any time within the patient's record. They were matched 1:1 to cases on age, sex, general practice, number of years of previous recorded history in the database, and calendar time (by assigning the index date of the case to his or her control). We applied the same exclusion criteria to controls as to cases.

\section{Definition and classification of thyroid disorders and their therapy}

We assessed whether cases and controls had a recorded Read-coded diagnosis of hypo- or hyperthyroidism prior to the index date. We further assessed drugs used to treat hypothyroidism (thyroid hormone replacement therapy, i.e., levothyroxine) or hyperthyroidism (thyroid hormone suppression therapy, i.e., carbimazole) by identifying prescriptions prior to the index date. We classified exposed patients into "current users" (last prescription recorded $<180$ days 
prior to the index date) and "past users" (last prescription recorded $\geq 180$ days prior to the index date). We determined a cutoff of 180 days for current use since these substances are prescribed for long-term use, and 90 days is the typical maximal length of a prescription in the UK. We further classified users according to duration of use, defining the number of prescriptions (1-9, 10-19, 20-29, and $\geq 30$ prescriptions) prior to the index date as proxy for treatment duration.

\section{Covariates}

Covariates in this study included BMI, categorized as (12.0 $18.4,18.5-24.9,25.0-29.9,30.0-59.0 \mathrm{~kg} / \mathrm{m}^{2}$, or unknown), smoking status (nonsmoker, current smoker, ex-smoker, or unknown), and alcohol consumption (never, current [1-9 units per week; 10-19 units per week; $\geq 20$ units per week], past, or unknown). We assessed whether cases and controls had a record (yes/no) of hypertension, diabetes mellitus, dyslipidemia, renal impairment, congestive heart failure, ischemic heart disease, or transient ischemic attack/stroke at any time prior to the index date. We assessed renal impairment by calculating the estimated GFR (eGFR) at or closest prior to the index date. We classified patients as having renal impairment if they had at least two increased measurements of serum creatinine that were $\geq 90$ days apart, resulting in two eGFR estimates of $<90 \mathrm{~mL} / \mathrm{min}$, or a Read code of chronic kidney disease (CKD), at any time prior to the index date. We used the Chronic Kidney Disease Epidemiology Collaboration (CKD-EPI) equation to calculate the eGFR. ${ }^{29,30}$ We further assessed ever versus never use of antihypertensive drugs such as angiotensin-converting enzyme inhibitors, angiotensin receptor blockers, diuretics (including thiazide diuretics, loop diuretics, and potassium-sparing diuretics), beta-blockers, calcium channel blockers, and nitrates as well as use of statins prior to the index date. Furthermore, we assessed the closest values of eGFR (no value, $<30 \mathrm{~mL} / \mathrm{min}, 30-60 \mathrm{~mL} / \mathrm{min}$, or 60-90 $\mathrm{mL} / \mathrm{min}$ ) recorded prior to the index date.

\section{Statistical analysis}

We conducted multivariate conditional logistic regression analyses to compare the exposure prevalence of hypo- or hyperthyroidism and thyroid hormone replacement or suppression therapy between cases and controls. We further stratified our analyses by thyroid hormone therapy (defined as the number of prescriptions of thyroid drugs prior to the index date), to assess potential effect modification by thyroid disease duration. We presented relative risk estimates as odds ratios (ORs) with $95 \%$ confidence intervals (CIs) and considered a two-sided $p$-value of $<0.05$ statistically significant. We conducted the statistical analysis using the software program SAS, Version 9.4 (SAS institute, Inc., Cary, NC, USA).

We adjusted our analyses for patient characteristics, comorbidities, or concomitant drug use in the multivariate analysis if these variables were predictor variables for gout known a priori from the literature. ${ }^{3}$ In all analyses, these were BMI, smoking status, and alcohol consumption. We additionally adjusted the analyses for lifestyle factors (alcohol consumption and smoking status) and concomitant diseases such as hypertension, congestive heart failure, ischemic heart disease, and renal impairment. We adjusted analyses for comedication use of diuretics, all other antihypertensive drugs assessed (including nitrates), and statins, recorded at any time prior to the index date.

\section{Sensitivity analyses}

In a first sensitivity analysis, we excluded patients with a history of renal impairment, congestive heart failure, or hypertension prior to the index date. In a second sensitivity analysis, we stratified the study population by the presence or absence of these comorbidities. In a third sensitivity analysis, we conducted the analysis in a subset of cases and their matched controls that were treated with nonsteroidal antiinflammatory drugs, colchicine, and/or corticosteroids within 7 days or with uricosuric/uricostatic drugs within 90 days prior to or after the index date. In a fourth sensitivity analysis, we restricted the study population to patients with incident thyroid disorders or use of thyroid therapy (patients with no diagnosis of thyroid disorder and no use of thyroid therapy within the first year of their registered history) to address potential prevalent user bias. In a fifth sensitivity analysis, we restricted the analysis to cases who were treated with thyroid hormone suppression therapy or nonpharmacological treatment only (i.e., surgical removal of parts of the gland, radiotherapy, or pharmacological intervention) prior to the index date.

\section{Stratified analyses and effect modification}

In a first analysis, we stratified the study population by sex to assess potential effect modification by sex on the association between thyroid disorders and gout. In a second analysis, we assessed the association between thyroid disorders and gout stratified by eGFR level (no value recorded, $<30 \mathrm{~mL} / \mathrm{min}$, $30-60 \mathrm{~mL} / \mathrm{min}$, or $60-90 \mathrm{~mL} / \mathrm{min}$ ).

\section{Results}

The study population encompassed 68,159 incident adult gout cases and the same number of matched controls. A total 
of $78.8 \%$ cases were male, resulting in a male-to-female ratio of 3.7 to 1 . Mean age ( \pm standard deviation) of cases was $58 \pm 15.8$ years, and female cases were older $(65.9 \pm$ 15.6 years $)$ than male cases $(56.6 \pm 15.0$ years $)$ at first gout diagnosis. Mean BMI at the closest general practitioner visit prior to the index date was $27.6 \pm 5.1 \mathrm{~kg} / \mathrm{m}^{2}$. Increasing BMI and increasing current alcohol consumption were associated with increasing ORs of gout. Further results are displayed in Table 1. Risk factors for gout that are known from the literature, such as hypertension, renal impairment, congestive heart failure, and ischemic heart disease, ${ }^{3}$ were all associated with an increased risk of incident gout in our study population. Current use of most antihypertensive drugs, except calcium channel blockers and nitrates, was associated with an increased OR of gout compared with never use of these drugs. Further results are displayed in Table 2.
There was no overall association between diagnosis of hypothyroidism and risk of gout (adjusted [adj] OR 1.12, 95\% CI 1.05-1.20) nor was hyperthyroidism associated with development of gout (adj OR 1.08, 95\% CI 0.95-1.22; Table 3). However, current short-term use of these drugs showed a tendency toward a small increase in risk: adj OR 1.54 (95\% CI 1.24-1.92) compared with nonuse. Thyroid hormone suppression therapy was not associated with gout. Further results are displayed in Table 4 . In the analysis of treatment duration, tests for trend were statistically significant for current short-term use (1-9 prescriptions prior to the index date) of thyroid hormone replacement therapy (data not shown).

\section{Sensitivity analyses}

There were no material differences from the main analysis in any of the five sensitivity analyses (Tables S1-S3).

Table I Demographics and characteristics of patients with gout and matched control patients

\begin{tabular}{|c|c|c|c|c|}
\hline Variable & $\begin{array}{l}\text { Cases, n (\%), } \\
\mathrm{N}=68,159\end{array}$ & $\begin{array}{l}\text { Controls, n (\%), } \\
\mathrm{N}=68,159\end{array}$ & OR crude $(95 \% \mathrm{Cl})$ & OR $\operatorname{adj} *(95 \% \mathrm{CI})$ \\
\hline \multicolumn{5}{|l|}{ Sex $^{\circ}$} \\
\hline Male & $53,736(78.8)$ & $53,736(78.8)$ & NA & NA \\
\hline Female & $14,423(2 \mid .2)$ & $14,423(2 \mid .2)$ & NA & NA \\
\hline \multicolumn{5}{|c|}{ Age group (years) ${ }^{\circ}$} \\
\hline$<29$ & $\mathrm{I}, 865(2.7)$ & $\mathrm{I}, 860(2.7)$ & NA & NA \\
\hline $30-39$ & $6,570(9.6)$ & $6,580(9.7)$ & NA & NA \\
\hline $40-49$ & $12,585(18.5)$ & $12,580(\mid 8.5)$ & NA & NA \\
\hline $50-59$ & $\mid 4,464(2 \mid .2)$ & $\mid 4,447(2 \mid .2)$ & NA & NA \\
\hline $60-69$ & $|4,05|(20.6)$ & $14,084(20.7)$ & NA & NA \\
\hline 70-79 & $12,034(17.7)$ & I2,044 (I7.7) & NA & NA \\
\hline$\geq 80$ & $6,590(9.7)$ & $6,564(9.6)$ & NA & NA \\
\hline \multicolumn{5}{|c|}{ Body mass index category $\left(\mathrm{kg} / \mathrm{m}^{2}\right)$} \\
\hline $12.0-18.4$ & $329(0.5)$ & $872(1.3)$ & $0.58(0.5 \mathrm{I}-0.66)$ & $0.67(0.58-0.77)$ \\
\hline $18.5-24.9$ & $|2,73|(\mid 8.7)$ & $20,633(30.3)$ & I (Reference) & I (Reference) \\
\hline $25.0-29.9$ & $24,778(36.4)$ & $21,573(31.7)$ & $1.92(1.86-1.98)$ & $1.74(1.68-1.79)$ \\
\hline $30.0-59.9$ & $19,298(28.3)$ & $9,962(14.6)$ & $3.32(3.21-3.44)$ & $2.76(2.66-2.87)$ \\
\hline Unknown & $11,023(16.2)$ & $15,119(22.2)$ & $1.10(1.06-1.14)$ & $1.46(1.39-1.52)$ \\
\hline \multicolumn{5}{|l|}{ Smoking status } \\
\hline Nonsmoker & $29,426(43.2)$ & $28,601(42.0)$ & I (Reference) & I (Reference) \\
\hline Current smoker & $10,703(15.7)$ & 13,899 (20.4) & $0.74(0.7 \mathrm{I}-0.76)$ & $0.75(0.72-0.77)$ \\
\hline Ex-smoker & $23,135(33.9)$ & $18,126(26.6)$ & $1.31(1.28-1.35)$ & $1.08(1.05-1.11)$ \\
\hline Unknown & $4,895(7.2)$ & $7,533(11.1)$ & $0.56(0.53-0.58)$ & $0.78(0.74-0.83)$ \\
\hline \multicolumn{5}{|c|}{ Alcohol consumption (units/week) } \\
\hline Never/ex & $8,143(12.0)$ & $9,616(14.1)$ & I (Reference) & I (Reference) \\
\hline Current unknown & $14,278(2 \mid .0)$ & $14,754(2 \mid .7)$ & $1.16(1.11-1.21)$ & $1.21(1.16-1.27)$ \\
\hline Current I-9 & $14,244(20.9)$ & $16,576(24.3)$ & $1.08(1.04-1.12)$ & $1.15(1.10-1.20)$ \\
\hline Current 10-19 & $9,768(14.3)$ & $8,090(11.9)$ & $1.57(1.50-1.64)$ & $1.72(1.63-1.80)$ \\
\hline Current $>20$ & $|4,29|(2 \mid .0)$ & $7,807(11.5)$ & $2.44(2.33-2.55)$ & $2.79(2.65-2.93)$ \\
\hline Unknown & $7,435(10.9)$ & $11,316(16.6)$ & $0.76(0.72-0.79)$ & $1.07(1.02-1.13)$ \\
\hline \multicolumn{5}{|c|}{ General practitioner visits last year } \\
\hline $0-2$ & $9,614(14.1)$ & $18,923(27.8)$ & & \\
\hline $3-4$ & $5,230(7.7)$ & $6,064(8.9)$ & $0.28(0.27-0.29)$ & $0.47(0.45-0.49)$ \\
\hline $5-9$ & $10,840(15.9)$ & $10,598(15.6)$ & $0.50(0.48-0.53)$ & $0.77(0.73-0.81)$ \\
\hline$\geq 10$ & $42,475(62.3)$ & $32,574(47.8)$ & $0.64(0.62-0.67)$ & $0.88(0.85-0.92)$ \\
\hline
\end{tabular}

Notes: ${ }^{\circ}$ Matching variables: age, sex, general practice, history on the database, and index date. ${ }^{\S} \mathrm{U}=10 \mathrm{~mL}$ of pure ethanol (8 $\mathrm{g}$ of ethanol). ${ }^{*}$ Adjusted for body mass index category, smoking status, alcohol consumption, hypertension, renal impairment, congestive heart failure, and ischemic heart disease/myocardial infarction. Abbreviations: adj, adjusted; $\mathrm{Cl}$, confidence interval; NA, not applicable; OR, odds ratio. 
Table 2 Comorbidities and comedication of patients with gout and matched control patients

\begin{tabular}{|c|c|c|c|c|}
\hline Comorbidities and comedication & Cases, $n(\%), N=68,159$ & Controls, $n$ (\%), $N=68,159$ & OR crude $(95 \% \mathrm{Cl})$ & OR $\operatorname{adj}(95 \% \mathrm{CI})$ \\
\hline \multicolumn{5}{|l|}{ Comorbidities $^{a}$} \\
\hline Hypertension & $27,379(40.2)$ & $16,423(24.1)$ & $2.54(2.47-2.6 \mathrm{I})$ & $1.86(1.80-1.92)$ \\
\hline Diabetes mellitus & $5,809(8.5)$ & $5,098(7.5)$ & $1.16(1.11-1.21)$ & $0.66(0.63-0.70)$ \\
\hline Dyslipidemia & $9,622(14.1)$ & $6,364(9.3)$ & $1.70(1.64-1.76)$ & $1.14(1.10-1.19)$ \\
\hline Renal impairment & $21,163(31.1)$ & $14,176(20.8)$ & $3.15(3.03-3.27)$ & $2.05(1.96-2.14)$ \\
\hline Congestive heart failure & $4,667(6.9)$ & $1,364(2.0)$ & $4.06(3.80-4.34)$ & $3.37(3.13-3.62)$ \\
\hline Ischemic heart disease & $10,309(15.1)$ & $6,572(9.6)$ & $1.80(1.74-1.87)$ & $1.23(1.18-1.28)$ \\
\hline Stroke/transient ischemic attack & $4,092(6.0)$ & $2,981(4.4)$ & $1.43(1.36-1.50)$ & $1.13(1.07-1.20)$ \\
\hline \multicolumn{5}{|l|}{ Comedication $^{\mathrm{b}}$} \\
\hline Angiotensin-converting enzyme inhibitors & $21,584(31.7)$ & $11,738(17.2)$ & $2.65(2.57-2.73)$ & $1.41(1.36-1.47)$ \\
\hline Angiotensin receptor blockers & $6,266(9.2)$ & $3,192(4.7)$ & $2.20(2.10-2.31)$ & $1.11(1.05-1.17)$ \\
\hline Loop diuretics & $11,366(16.7)$ & $4,664(6.8)$ & $3.35(3.21-3.49)$ & $2.29(2.18-2.4 I)$ \\
\hline Thiazide diuretics & $17,672(25.9)$ & $9,879(14.5)$ & $2.37(2.30-2.45)$ & $1.63(1.57-1.69)$ \\
\hline Potassium-sparing diuretics & $2,862(4.2)$ & $863(1.3)$ & $3.57(3.29-3.86)$ & $1.69(1.54-1.85)$ \\
\hline Beta-blockers & $22,758(33.4)$ & $14,240(20.9)$ & $2.08(2.02-2.14)$ & $1.40(1.35-1.44)$ \\
\hline Calcium channel blockers & $17,085(25.1)$ & $10,919(16.0)$ & $1.93(1.88-1.99)$ & $1.00(0.96-1.04)$ \\
\hline Nitrates & $9,332(13.7)$ & $6,144(9.0)$ & $1.70(1.64-1.76)$ & $1.02(0.97-1.07)$ \\
\hline Statins & $18,080(26.5)$ & $|2,45|(\mid 8.3)$ & $1.88(1.83-1.94)$ & $1.08(1.04-1.13)$ \\
\hline
\end{tabular}

Notes: aAdjusted for body mass index category, smoking status, alcohol consumption, and concomitant diseases such as hypertension, renal impairment, congestive heart failure, and ischemic heart disease/myocardial infarction. ${ }^{b}$ Adjusted for body mass index category, smoking status, alcohol consumption, and concomitant prescriptions of angiotensin-converting-enzyme inhibitors, angiotensin receptor blockers, loop diuretics, thiazide diuretics, potassium-sparing diuretics, beta-blockers, calcium channel blockers, nitrates, and statins.

Abbreviations: adj, adjusted; $\mathrm{Cl}$, confidence interval; OR, odds ratio

Table 3 Thyroid diseases of patients with gout and matched control patients stratified by timing

\begin{tabular}{|c|c|c|c|c|}
\hline Thyroid disorders & Cases (\%), N=68, I59 & Controls, n (\%), N=68, I59 & OR crude $(95 \% \mathrm{Cl})$ & OR $\operatorname{adj}^{a}(95 \% \mathrm{Cl})$ \\
\hline \multicolumn{5}{|l|}{ Hypothyroidism } \\
\hline No & $65,202(95.7)$ & $65,951(96.8)$ & I (Reference) & I (Reference) \\
\hline Yes & $2,957(4.3)$ & $2,208(3.2)$ & $1.38(1.30-1.46)$ & $1.12(1.05-1.20)$ \\
\hline \multicolumn{5}{|l|}{ Hyperthyroidism } \\
\hline No & $67,465(99.0)$ & $67,582(99.2)$ & I (Reference) & I (Reference) \\
\hline Yes & $694(1.0)$ & $577(0.9)$ & $1.21(1.08-1.35)$ & $1.08(0.95-1.22)$ \\
\hline
\end{tabular}

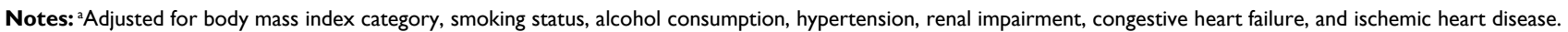
Abbreviations: adj, adjusted; $\mathrm{Cl}$, confidence interval; OR, odds ratioadj.

\section{Stratified analyses and effect modification}

There were no material differences from the main analysis when stratified by sex (Table S4). In patients who had an impaired eGFR at the closest general practitioner visit prior to the index date the effect in those with lower eGFR was statistically different from those with better renal function. However, hypo- and hyperthyroidism were both not associated with gout (Table 5). Only patients without recorded eGFR prior to the index date were at slightly increased risk for incident gout in patients with hypo- or hyperthyroidism (Table 5).

\section{Discussion}

In this large population-based study using data from the UKbased CPRD, we explored the risk of incident gout in association with previously diagnosed hypo- or hyperthyroidism and with their pharmacological therapy. While there was no clinically relevant overall association of hypo- or hyperthyroidism and risk of gout, there was a small suggestion that current exposure to new onset hypothyroidism and its short-term treatment may increase the risk compared with no hypothyroidism or hormone replacement therapy. Neither hyperthyroidism nor its therapy was associated with an increased risk of gout.

Our results in newly diagnosed and treated hypothyroidism are consistent with previous studies that reported a high prevalence of hyperuricemia in patients with hypothyroidism. ${ }^{11,12}$ We found no association between hyperthyroidism and gout despite available evidence from previous studies that hyperuricemia was present in patients with hyperthyroidism. ${ }^{10-12}$ The tendency toward a positive association between hypothyroidism and gout might be explained by the fact that thyroid hormones influence serum urate levels through regulation of GFR. ${ }^{13}$ Nevertheless, if this association was only explained by renal impairment, patients with hypothyroidism whose kidney function was normal should not be at an increased risk of developing incident gout. Our analysis stratified by renal function, however, suggests 
Table 4 Thyroid disease therapies of patients with gout and matched control patients stratified by timing and duration

\begin{tabular}{|c|c|c|c|c|}
\hline $\begin{array}{l}\text { Thyroid disorder } \\
\text { therapies }\end{array}$ & Cases, n (\%), N=68, I 59 & Controls, n (\%), N=68, 159 & OR crude $(95 \% \mathrm{Cl})$ & OR $\operatorname{adj}^{a}(95 \% \mathrm{Cl})$ \\
\hline \multicolumn{5}{|c|}{ Treatment with thyroid HRT } \\
\hline Non-use & $65,032(95.4)$ & $65,846(96.6)$ & I (Reference) & I (Reference) \\
\hline Overall use & $3,127(4.6)$ & $2,313(3.4)$ & $1.40(1.32-1.48)$ & $1.15(1.08-1.23)$ \\
\hline \multicolumn{5}{|l|}{ Number of $\mathrm{Rx}$} \\
\hline I-9 Rx & $406(0.6)$ & $247(0.4)$ & $1.68(1.43-1.97)$ & $1.39(1.16-1.66)$ \\
\hline $10-19 R x$ & $290(0.4)$ & $244(0.4)$ & $1.22(1.03-1.45)$ & $1.00(0.82-1.21)$ \\
\hline $20-29 \mathrm{Rx}$ & $275(0.4)$ & $219(0.3)$ & $1.30(1.09-1.55)$ & I.II (0.9I-I.37) \\
\hline$\geq 30 \mathrm{Rx}$ & $2,156(3.2)$ & $\mathrm{I}, 603(2.4)$ & $1.40(1.30-1.49)$ & $1.15(1.06-1.24)$ \\
\hline \multicolumn{5}{|l|}{ Current use $\mathrm{b}^{\mathrm{b}}$} \\
\hline Overall & $2,964(4.4)$ & $2,146(3.2)$ & $1.43(1.35-1.5 I)$ & $1.17(1.09-1.25)$ \\
\hline I-9 Rx & $30 \mathrm{I}(0.4)$ & $154(0.2)$ & $1.99(1.64-2.42)$ & $1.54(1.24-1.92)$ \\
\hline $10-19 \mathrm{Rx}$ & $260(0.4)$ & $219(0.3)$ & $1.21(1.01-1.45)$ & $0.98(0.80-1.21)$ \\
\hline $20-29 R x$ & $265(0.4)$ & $206(0.3)$ & $1.33(1.11-1.60)$ & $1.15(0.93-1.42)$ \\
\hline$\geq 30 \mathrm{Rx}$ & $2,138(3.1)$ & $\mathrm{I}, 567(2.3)$ & $1.4 \mid(|.32-| .5 I)$ & $1.16(1.07-1.25)$ \\
\hline \multicolumn{5}{|l|}{ Past use ${ }^{c}$} \\
\hline Overall & $163(0.2)$ & $167(0.3)$ & $1.01(0.82-1.26)$ & $0.94(0.73-1.20)$ \\
\hline \multicolumn{5}{|c|}{ Treatment with thyroid HST } \\
\hline Nonuse & 67,817 (99.5) & $67,870(99.6)$ & I (Reference) & I (Reference) \\
\hline Overall use & $342(0.5)$ & $289(0.4)$ & $1.19(1.01-1.39)$ & $0.94(0.78-1.12)$ \\
\hline \multicolumn{5}{|l|}{ Number of Rx } \\
\hline I-9 Rx & $215(0.3)$ & $165(0.2)$ & $1.30(1.06-1.60)$ & $1.03(0.8|-| .3 \mid)$ \\
\hline $10-19 \mathrm{Rx}$ & $70(0.1)$ & $64(0.1)$ & $1.10(0.78-1.54)$ & $0.83(0.56-1.22)$ \\
\hline $20-29 R x$ & $16(0.0)$ & $22(0.0)$ & $0.73(0.38-1.39)$ & $0.65(0.30-1.39)$ \\
\hline$\geq 30 \mathrm{Rx}$ & $4 I(0.1)$ & $38(0.1)$ & $1.08(0.69-1.68)$ & $0.87(0.53-1.45)$ \\
\hline \multicolumn{5}{|l|}{ Current use ${ }^{d}$} \\
\hline Overall & $82(0.1)$ & $80(0.1)$ & $1.03(0.75-1.40)$ & $0.82(0.57-1.17)$ \\
\hline I-9 Rx & $34(0.1)$ & $29(0.0)$ & $1.17(0.7 \mid-1.92)$ & $0.91(0.50-1.63)$ \\
\hline $10-19 \mathrm{Rx}$ & $17(0.0)$ & $12(0.0)$ & $1.42(0.68-2.98)$ & $0.90(0.4 I-2.0 I)$ \\
\hline $20-29 R x$ & $9(0.0)$ & $10(0.0)$ & $0.90(0.37-2.22)$ & $1.16(0.43-3.17)$ \\
\hline$\geq 30 \mathrm{Rx}$ & $22(0.0)$ & $29(0.0)$ & $0.76(0.44-1.32)$ & $0.59(0.3 \mid-1.12)$ \\
\hline \multicolumn{5}{|l|}{ Past use $\mathrm{e}^{\mathrm{e}}$} \\
\hline Overall & $260(0.4)$ & $209(0.3)$ & $1.25(1.04-1.50)$ & $0.98(0.79-1.22)$ \\
\hline
\end{tabular}

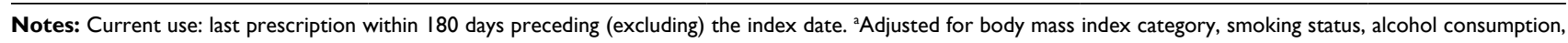
angiotensin-converting enzyme inhibitors, angiotensin receptor blockers, loop diuretics, thiazide diuretics, potassium-sparing diuretics, beta-blockers, calcium channel blockers, nitrates, and statins. 'Last prescription of thyroid HRT recorded $<180$ days prior to (excluding) the index date. 'Last prescription of thyroid HRT recorded

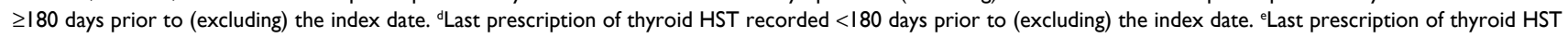
recorded $\geq 180$ days prior to (excluding) the index date.

Abbreviations: adj, adjusted; $\mathrm{Cl}$, confidence interval; HRT, hormone replacement therapy; HST, hormone suppression therapy; OR, odds ratio; Rx: prescriptions.

that patients with hypothyroidism or hyperthyroidism who had no recorded renal impairment were still at a slightly increased risk of developing gout compared to patients without thyroid diseases and no recorded renal impairment. This observation might be explained by other effects of hypo- and hyperthyroidism on kidney function that cannot be detected by measuring GFR, such as metabolic or cardiovascular effects. ${ }^{13}$ However, the influence of renal impairment within this subpopulation of patients without eGFR is beyond the scope of this study. Even if patients with recently diagnosed thyroid disease might have seen more often their physician, gouty arthritis is a very painful clinical condition and is most likely not diagnosed routinely or by chance. It is more likely that patients see their general practitioner because of acute joint pain than that they just wait for a follow-up consultation. It may be possible that patients who just started their treatment of hypothyroidism may be at greater risk of gout because a potential protective effect of thyroid hormone replacement therapy did not yet become active.

This large population-based study has several strengths. First, we were able to study a large number of cases with incident gout in a well-established and validated primary care database. Second, the diagnosis of gout has been validated, ${ }^{27}$ and similar case definitions for incident gout have been used by other authors. ${ }^{3,23-28}$ Third, we could study many cases with thyroid disorders and conduct various sensitivity analyses to address in-depth the potential role of thyroid hormone replacement or suppression therapy in the risk of incident gout. Fourth, unlike former studies, ${ }^{7-12}$ we could 
Table 5 Thyroid diseases of patients with gout and matched control patients stratified by GFR

\begin{tabular}{|c|c|c|c|c|}
\hline Glomerular filtration rate & Cases, n (\%), N=68, 159 & Controls, n (\%), N=68, 159 & OR crude $(95 \% \mathrm{Cl})$ & OR adj* $(95 \% \mathrm{Cl})$ \\
\hline \multicolumn{5}{|c|}{ Value of last eGFR prior to the index date } \\
\hline \multicolumn{5}{|c|}{ Overall population } \\
\hline No value & $4 \mathrm{I}, 340(60.7)$ & 48,440 (7I.I) & $0.38(0.36-0.39)$ & $0.66(0.63-0.69)$ \\
\hline$<30 \mathrm{~mL} / \mathrm{min}$ & $1,119(1.6)$ & $194(0.3)$ & $10.99(9.18-13.16)$ & $7.76(6.41-9.40)$ \\
\hline $30-60 \mathrm{~mL} / \mathrm{min}$ & $5,306(7.8)$ & $2,972(4.4)$ & $2.90(2.69-3.12)$ & $2.43(2.24-2.63)$ \\
\hline $60-90 \mathrm{~mL} / \mathrm{min}$ & $20,394(29.9)$ & $16,553(24.3)$ & I (Reference) & I (Reference) \\
\hline \multicolumn{5}{|l|}{ Hypothyroidism } \\
\hline Hypothyroidism, no value & $946(1.4)$ & $850(1.3)$ & $1.39(1.26-1.53)$ & $1.25(1.09-1.35)$ \\
\hline No hypothyroidism, no value & $40,394(59.3)$ & $47,590(69.8)$ & I (Reference) & I (Reference) \\
\hline Hypothyroidism, $<30$ mL/min & $184(0.3)$ & $34(0.1)$ & $0.85(0.55-|.3|)$ & $0.79(0.50-1.24)$ \\
\hline No hypothyroidism, $<30 \mathrm{~mL} / \mathrm{min}$ & $935(1.4)$ & $160(0.2)$ & I (Reference) & I (Reference) \\
\hline Hypothyroidism, $30-59$ mL/min & $735(1.1)$ & $384(0.6)$ & $1.14(0.99-1.32)$ & $1.03(0.88-1.21)$ \\
\hline No hypothyroidism, $30-59 \mathrm{~mL} / \mathrm{min}$ & $4,571(6.7)$ & $2,588(3.8)$ & I (Reference) & I (Reference) \\
\hline Hypothyroidism, $60-90 \mathrm{~mL} / \mathrm{min}$ & $1,092(1.6)$ & $940(1.4)$ & $1.07(0.97-1.18)$ & $1.01(0.90-1.12)$ \\
\hline No hypothyroidism, $60-90 \mathrm{~mL} / \mathrm{min}$ & $19,302(28.3)$ & $15,613(22.9)$ & I (Reference) & I (Reference) \\
\hline \multicolumn{5}{|l|}{ Hyperthyroidism } \\
\hline Hyperthyroidism, no value & $946(0.4)$ & $283(0.4)$ & $1.17(0.98-1.39)$ & $1.25(1.03-1.52)$ \\
\hline No hyperthyroidism, no value & $40,394(60.3)$ & $48,157(70.6)$ & I (Reference) & I (Reference) \\
\hline Hyperthyroidism, $<30 \mathrm{~mL} / \mathrm{min}$ & $184(0.1)$ & $5(0.01)$ & $\mathrm{I} . \mathrm{II}(0.4 \mathrm{I}-3.0 \mathrm{I})$ & $1.02(0.36-2.93)$ \\
\hline No hyperthyroidism, $<30 \mathrm{~mL} / \mathrm{min}$ & $\mathrm{I}, 087(\mathrm{I} .6)$ & $189(0.3)$ & I (Reference) & I (Reference) \\
\hline Hyperthyroidism, $30-59 \mathrm{~mL} / \mathrm{min}$ & $144(0.2)$ & $82(0.1)$ & $0.98(0.72-1.32)$ & $0.88(0.63-1.23)$ \\
\hline No hyperthyroidism, $30-59 \mathrm{~mL} / \mathrm{min}$ & $5,162(7.6)$ & $2,890(4.2)$ & I (Reference) & I (Reference) \\
\hline Hyperthyroidism, $60-90 \mathrm{~mL} / \mathrm{min}$ & $259(0.4)$ & $207(0.3)$ & $1.09(0.90-1.33)$ & $0.94(0.75-1.17)$ \\
\hline No hyperthyroidism, $60-90 \mathrm{~mL} / \mathrm{min}$ & $20,135(29.5)$ & $16,346(24.0)$ & I (Reference) & I (Reference) \\
\hline
\end{tabular}

Notes: The reference is in each category the same kidney function category without the respective thyroid disease, eg, hypothyroidism $<30 \mathrm{~mL} / \mathrm{min}$ compared to no hypothyroidism $<30 \mathrm{~mL} / \mathrm{min}$. *Adjusted for body mass index category, smoking status, alcohol consumption, hypertension, renal failure, congestive heart failure, and ischemic heart disease.

Abbreviations: adj, adjusted; $\mathrm{Cl}$, confidence interval; eGFR, estimated glomerular filtration rate; OR, odds ratio.

adjust our analyses for important potential confounders such as age and sex, by matching, and important lifestyle factors, comorbidities, or concomitant drug therapy, by including them in the multivariable regression models. Fifth, recall bias was not an issue in this study as information on diseases and drug exposure was prospectively entered in the CPRD in the absence of any study hypothesis. Finally, exclusion of all patients with $<3$ years of recorded history in the database prior to the index date reduced the likelihood of including prevalent rather than incident gout patients.

Some limitations of our study must be acknowledged. Although the diagnosis of gout has been validated and found to be well documented in the $\mathrm{CPRD},{ }^{27}$ there may still be some outcome misclassification. Gout diagnoses are often made based on clinical presentation and are rarely confirmed in routine clinical practice by analysis of aspirated joint fluid for evidence of urate crystals. To minimize misclassification, we only included gout patients who had no differential diagnoses of gout such as osteoarthritis, septic arthritis, hemochromatosis, and rheumatoid arthritis recorded at any time within their patient record. We were not able to disentangle effects of the underlying thyroid disorder from effects of thyroid hormone therapy on the risk of gout because hypo- and hyperthyroidism are treated with thyroid hormone replacement or suppression therapy. A further limitation was that GFR measurements were not available for all patients in the study. This is a common problem when dealing with laboratory values or biomarkers that are an important part of diagnostic assessments. Residual confounding by indication by CKD and congestive heart failure which are causally linked to development of hyperuricemia, cannot be excluded in the present study despite every effort to minimize such a bias. We adjusted for use of drugs indicated for treatment of these comorbidities, and we stratified our analyses by the most important chronic comorbidities, namely arterial hypertension, congestive heart failure, and renal impairment.

We did not adjust for all potential risk factors for gout, since, for example, dietary habits or physical activities ${ }^{2,31}$ are not routinely recorded in the CPRD. However, we adjusted for BMI, a factor that is related to physical activity and dietary habits. Furthermore, we were not able to take race/ethnicity into account when calculating eGFR with the CKD-EPI formula because ethnicity information is not consistently available in the CPRD. However, since most individuals living in 
the UK are Caucasian, ${ }^{32}$ our results are representative of large portion of the UK population. Finally, we could not address potential confounding by socioeconomic status; however, we partially controlled for this potential confounder by matching cases and controls from the same general practice, since it is likely that patients from the same neighborhood see the same general practitioner.

\section{Conclusion}

This large observational study does not provide evidence that hypothyroidism, irrespective of its treatment, and hyperthyroidism, are associated with a clinically relevant increased risk of developing incident gout. There may be a small increased risk of gout in patients with newly diagnosed and treated hypothyroidism.

\section{Acknowledgments}

The authors thank Pascal Egger for programming and technical support. This study was supported by an unconditional grant by the Margot and Erich Goldschmidt \& Peter René Jacobson Foundation, Switzerland.

\section{Disclosure}

The authors report no conflicts of interest in this work.

\section{References}

1. Riches PL, Wright AF, Ralston SH. Recent insights into the pathogenesis of hyperuricaemia and gout. Hum Mol Genet. 2009;18(R2): R177-R184.

2. Choi HK, Mount DB, Reginato AM. Pathogenesis of gout. Ann Intern Med. 2005;143(7):499-516.

3. Mikuls TR, Farrar JT, Bilker WB, Fernandes S, Schumacher HR, Saag KG. Gout epidemiology: results from the UK General Practice Research Database, 1990-1999. Ann Rheum Dis. 2005;64(2):267-272.

4. Annemans L, Spaepen E, Gaskin M, et al. Gout in the UK and Germany: prevalence, comorbidities and management in general practice 20002005. Ann Rheum Dis. 2008;67(7):960-966.

5. Harrold LR, Yood RA, Mikuls TR, et al. Sex differences in gout epidemiology: evaluation and treatment. Ann Rheum Dis. 2006;65(10): 1368-1372.

6. Dirken-Heukensfeldt KJ, Teunissen T, van de Lisdonk E, Lagro-Janssen A. "Clinical features of women with gout arthritis." A systematic review. Clin Rheumatol. 2010;29(6):575-582.

7. Kuzell W, Schaffarzick R, Naugler W, et al. Some observations on 520 gouty patients. J Chronic Dis. 1955;2(6):645-669.

8. Durward W. Letter: gout and hypothyroidism in males. Arthritis Rheum. 1976;19(1):123.

9. Erickson AR, Enzenauer RJ, Nordstrom DM, Merenich JA. The prevalence of hypothyroidism in gout. Am J Med. 1994;97(3):231-234.

10. See L-C, Kuo C-F, Yu K-H, et al. Hyperthyroid and hypothyroid status was strongly associated with gout and weakly associated with hyperuricaemia. PLoS One. 2014;9(12):e114579.
11. Giordano N, Santacroce C, Mattii G, Geraci S, Amendola A, Gennari C. Hyperuricemia and gout in thyroid endocrine disorders. Clin Exp Rheumatol. 2001;19(6):661-665.

12. Sato A, Shirota T, Shinoda T, et al. Hyperuricemia in patients with hyperthyroidism due to Graves' disease. Metabolism. 1995;44(2): 207-211.

13. Mariani LH, Berns JS. The renal manifestations of thyroid disease. J Am Soc Nephrol. 2011;23(1):22-26.

14. Saini V, Yadav A, Arora MK, Arora S, Singh R, Bhattacharjee J. Correlation of creatinine with TSH levels in overt hypothyroidism - a requirement for monitoring of renal function in hypothyroid patients? Clin Biochem. 2012;45(3):212-214.

15. Herrett E, Gallagher AM, Bhaskaran K, et al. Data resource profile: clinical practice research datalink (CPRD). Int J Epidemiol. 2015;44(3):827-836.

16. Jick H. A database worth saving. Lancet. 1997;350(9084):1045-1046.

17. Walley T, Mantgani A. The UK General Practice Research Database. Lancet. 1997;350(9084):1097-1099.

18. Khan NF, Harrison SE, Rose PW. Validity of diagnostic coding within the General Practice Research Database: a systematic review. Br J Gen Pract. 2010;60(572):e128-e136.

19. Jick H, Jick SS, Derby LE. Validation of information recorded on general practitioner based computerised data resource in the United Kingdom. BMJ. 1991;302(6779):766-768.

20. Jick SS, Kaye JA, Vasilakis-Scaramozza C, et al. Validity of the general practice research database. Pharmacotherapy. 2003;23(5):686-689.

21. Herrett E, Thomas SL, Schoonen WM, Smeeth L, Hall AJ. Validation and validity of diagnoses in the General Practice Research Database: a systematic review. Br J Clin Pharmacol. 2010;69(1):4-14.

22. Jick H, Jick SS, Derby LE, Terris BZ. Further validation of information recorded on general practitioner based computerised data resource in the United Kingdom. Pharmacoepidemiol Drug Saf. 1992;1: 347-349.

23. Bruderer S, Bodmer M, Jick SS, Meier CR. Use of diuretics and risk of incident gout: a population-based case-control study.[Erratum appears in Arthritis Rheumatol. 2014 Feb;66(2):427]. Arthritis Rheumatol. 2014;66(1):185-196.

24. Bruderer SG, Bodmer M, Jick SS, Meier CR. Poorly controlled type 2 diabetes mellitus is associated with a decreased risk of incident gout: a population-based case-control study. Ann Rheum Dis. 2015;74(9):1651-1658.

25. Bruderer SG, Bodmer M, Jick SS, Meier CR. Association of hormone therapy and incident gout: population-based case-control study. Menopause. 2015;22(12):1-8.

26. Kuo C, Grainge MJ, Mallen C, Zhang W, Doherty M. Rising burden of gout in the UK but continuing suboptimal management: a nationwide population study. Ann Rheum Dis. 2015;74(4):661-667.

27. Meier CR, Jick H. Omeprazole, other antiulcer drugs and newly diagnosed gout. Br J Clin Pharmacol. 1997;44(2):175-178.

28. Alonso A, Rodriquez LA, Logroscino G, Hernan MA. Gout and risk of Parkinson disease: a prospective study. Neurology. 2007;69(17):1696-1700.

29. National Kidney Foundation. K/DOQI clinical practice guidelines for chronic kidney disease: evaluation, classification, and stratification. $\mathrm{Am}$ J Kidney Dis. 2002;39(2 suppl 1):S1-S26.

30. Levey AS, Stevens LA, Schmid CH, et al. A new equation to estimate glomerular filtration rate. Ann Intern Med. 2009;150(9):604-612.

31. Choi HK, Atkinson K, Karlson EW, Curhan G. Obesity, weight change, hypertension, diuretic use, and risk of gout in men: the Health Professionals Follow-up Study. Arch Intern Med. 2005;165(7):742-748.

32. 2011 UKC. United Kingdom Population by Ethnic Group. Newport, UK: Office for National Statistics; 2011. 


\section{Supplementary materials}

Table SI Sensitivity analysis I: patients without hypertension, congestive heart failure, or renal impairment prior to the index date

\begin{tabular}{|c|c|c|c|c|}
\hline Thyroid disorders & Cases, n (\%), N=33, 191 & Controls, $n(\%), N=45,363$ & OR crude $(95 \% \mathrm{Cl})$ & OR $\operatorname{adj} *(95 \% \mathrm{CI})$ \\
\hline \multicolumn{5}{|l|}{ Hypothyroidism } \\
\hline No & $32,604(98.2)$ & $44,597(98.3)$ & I (Reference) & I (Reference) \\
\hline Yes & $587(1.8)$ & $766(1.7)$ & $1.29(1.12-1.50)$ & $1.12(0.95-1.3 \mathrm{I})$ \\
\hline Current diagnosis & $67(0.2)$ & $69(0.2)$ & $1.40(0.91-2.17)$ & $1.13(0.7 I-1.82)$ \\
\hline Past diagnosis & $520(1.6)$ & $697(1.5)$ & $1.28(1.10-1.50)$ & $1.12(0.94-1.32)$ \\
\hline \multicolumn{5}{|l|}{ Hyperthyroidism } \\
\hline No & $33,028(99.5)$ & $45,116(99.5)$ & I (Reference) & I (Reference) \\
\hline Yes & $163(0.5)$ & $247(0.5)$ & $1.25(0.96-1.63)$ & $0.73(0.58-0.91)$ \\
\hline Current diagnosis & $8(0.0)$ & $18(0.0)$ & $0.65(0.25-1.67)$ & $0.70(0.24-2.06)$ \\
\hline Past diagnosis & $155(0.5)$ & $229(0.5)$ & $1.32(1.00-1.75)$ & $1.35(1.00-1.83)$ \\
\hline
\end{tabular}

Notes: Current diagnosis: last diagnosis within 180 days prior to (excluding) index date. *Adjusted for body mass index category, smoking status, alcohol consumption, and ischemic heart disease.

Abbreviations: adj, adjusted; $\mathrm{Cl}$, confidence interval; OR, odds ratio.

Table S2 Sensitivity analysis 2: stratified by hypertension, congestive heart failure, and renal impairment

\begin{tabular}{|c|c|c|c|c|}
\hline Stratification variable & Cases, $\mathrm{n}(\%), \mathrm{N}=68,159$ & Controls, $n$ (\%), N=68, I59 & OR crude $(95 \% \mathrm{Cl})$ & OR $\operatorname{adj} *(95 \% \mathrm{Cl})$ \\
\hline \multicolumn{5}{|l|}{ Hypertension } \\
\hline \multicolumn{5}{|l|}{ Hypothyroidism } \\
\hline \multicolumn{5}{|l|}{ Without hypertension } \\
\hline No & $39,564(58.1)$ & $50,47 I(74.1)$ & I (Reference) & I (Reference) \\
\hline Yes & $1,216(1.8)$ & $1,265(1.9)$ & $1.46(1.34-1.59)$ & $1.15(1.05-1.26)$ \\
\hline \multicolumn{5}{|l|}{ With hypertension } \\
\hline No & $25,638(37.6)$ & $15,480(22.7)$ & I (Reference) & I (Reference) \\
\hline Yes & $\mathrm{I}, 74 \mathrm{I}(2.6)$ & $943(1.4)$ & $1.24(1.14-1.35)$ & $1.10(1.00-1.20)$ \\
\hline \multicolumn{5}{|l|}{ Hyperthyroidism } \\
\hline \multicolumn{5}{|l|}{ Without hypertension } \\
\hline No & $40,484(59.4)$ & $51,390(75.4)$ & I (Reference) & I (Reference) \\
\hline Yes & $296(0.4)$ & $346(0.5)$ & $1.25(1.07-1.47)$ & $1.14(0.96-1.36)$ \\
\hline \multicolumn{5}{|l|}{ With hypertension } \\
\hline No & $26,98 I(39.6)$ & $16,192(23.8)$ & I (Reference) & I (Reference) \\
\hline Yes & $398(0.6)$ & $231(0.3)$ & $1.07(0.91-1.27)$ & I.0I $(0.84-1.2 I)$ \\
\hline \multicolumn{5}{|l|}{ Congestive heart failure } \\
\hline \multicolumn{5}{|c|}{ Hypothyroidism } \\
\hline \multicolumn{5}{|c|}{ Without congestive heart failure } \\
\hline No & $60,992(89.5)$ & $64,698(94.9)$ & I (Reference) & I (Reference) \\
\hline Yes & $2,500(3.7)$ & $2,097(3.1)$ & $1.32(1.25-1.4 \mid)$ & $1.13(1.05-1.21)$ \\
\hline \multicolumn{5}{|c|}{ With congestive heart failure } \\
\hline No & $4,210(6.2)$ & $\mathrm{I}, 253(\mathrm{I} .8)$ & I (Reference) & I (Reference) \\
\hline Yes & $457(0.7)$ & $\mathrm{III}(0.2)$ & $1.19(0.95-1.48)$ & $1.10(0.87-1.40)$ \\
\hline \multicolumn{5}{|c|}{ Hyperthyroidism } \\
\hline \multicolumn{5}{|c|}{ Without congestive heart failure } \\
\hline No & $62,948(92.4)$ & $66,260(97.2)$ & I (Reference) & I (Reference) \\
\hline Yes & $544(0.8)$ & $535(0.8)$ & I.II (0.98-I.26) & $1.09(0.95-1.25)$ \\
\hline \multicolumn{5}{|c|}{ With congestive heart failure } \\
\hline No & $4,517(6.6)$ & $\mathrm{I}, 322(\mathrm{I} .9)$ & I (Reference) & I (Reference) \\
\hline Yes & $150(0.2)$ & $42(0.1)$ & $0.98(0.69-1.39)$ & $0.97(0.66-I .4 I)$ \\
\hline \multicolumn{5}{|l|}{ Renal impairment } \\
\hline \multicolumn{5}{|l|}{ Hypothyroidism } \\
\hline \multicolumn{5}{|l|}{ Without renal impairment } \\
\hline No & $45,839(67.3)$ & $52,886(77.6)$ & I (Reference) & I (Reference) \\
\hline Yes & $\mathrm{I}, 157(1.7)$ & $\mathrm{I}, 097(1.6)$ & $1.28(1.18-1.40)$ & $1.14(1.04-1.25)$ \\
\hline \multicolumn{5}{|l|}{ With renal impairment } \\
\hline No & $19,363(28.4)$ & 13,065 (19.2) & I (Reference) & I (Reference) \\
\hline Yes & $1,800(2.6)$ & $\mathrm{I}, \mathrm{II} \mathrm{I}(\mathrm{I} .6)$ & $1.15(1.06-1.25)$ & 1.11 (1.02-1.22) \\
\hline
\end{tabular}


Table S2 (Continued)

\begin{tabular}{|c|c|c|c|c|}
\hline & Cases, n (\%), N=68, I 59 & Controls, n (\%), N=68, I 59 & OR crude $(95 \% \mathrm{CI})$ & OR $\operatorname{adj} *(95 \%$ CI) \\
\hline \multicolumn{5}{|c|}{ Hyperthyroidism } \\
\hline \multicolumn{5}{|c|}{ Without renal impairment } \\
\hline No & $46,684(68.5)$ & $53,635(78.7)$ & I (Reference) & I (Reference) \\
\hline Yes & $312(0.5)$ & $348(0.5)$ & $\mathrm{I} .08(0.92-1.26)$ & I.I $3(0.95-1.34)$ \\
\hline \multicolumn{5}{|c|}{ With renal impairment } \\
\hline No & $20,78 \mid(30.5)$ & $13,947(20.5)$ & I (Reference) & I (Reference) \\
\hline Yes & $382(0.6)$ & $229(0.3)$ & I.I3 (0.95-I.35) & $\mathrm{I} .02(0.84-\mathrm{I} .23)$ \\
\hline
\end{tabular}

Notes: *Adjusted for body mass index category, smoking status, alcohol consumption, hypertension, and renal failure.

Abbreviations: adj, adjusted; $\mathrm{Cl}$, confidence interval; OR, odds ratio.

Table S3 Sensitivity analysis 3: patients with treated gout compared with matched controls

\begin{tabular}{|c|c|c|c|c|}
\hline Thyroid disorders & Cases, n (\%), N=45,466 & Controls, $n(\%), N=45,466$ & OR crude $(95 \% \mathrm{Cl})$ & OR adj* $(95 \% \mathrm{Cl})$ \\
\hline \multicolumn{5}{|l|}{ Hypothyroidism } \\
\hline No & $43,478(95.6)$ & $43,987(96.8)$ & I (Reference) & I (Reference) \\
\hline Yes & $\mathrm{I}, 988(4.4)$ & $\mathrm{I}, 479(3.3)$ & $1.39(1.29-1.49)$ & $1.10(1.02-1.20)$ \\
\hline Current diagnosis & $165(0.4)$ & $110(0.2)$ & $1.54(1.21-1.96)$ & $1.34(1.01-1.77)$ \\
\hline Past diagnosis & $\mathrm{I}, 823(4.0)$ & $1,369(3.0)$ & $1.37(1.28-1.48)$ & $1.09(1.00-1.18)$ \\
\hline \multicolumn{5}{|l|}{ Hyperthyroidism } \\
\hline No & $44,978(98.9)$ & $45,063(99.1)$ & I (Reference) & I (Reference) \\
\hline Yes & $488(I .1)$ & $403(0.9)$ & $1.21(1.06-1.39)$ & $\mathrm{I} .03(0.88-1.2 \mathrm{I})$ \\
\hline Current diagnosis & $31(0.1)$ & $24(0.1)$ & $1.29(0.76-2.20)$ & $0.92(0.49-1.70)$ \\
\hline Past diagnosis & $457(1.0)$ & $379(0.8)$ & $1.21(1.05-1.39)$ & $1.04(0.88-1.22)$ \\
\hline
\end{tabular}

Notes: Current diagnosis: last diagnosis within 180 days prior to (excluding) index date. *Adjusted for body mass index category, smoking status, alcohol consumption, hypertension, renal failure, congestive heart failure, and ischemic heart disease.

Abbreviations: adj, adjusted; $\mathrm{Cl}$, confidence interval; OR, odds ratio.

Table S4 Test for effect modification analysis I: stratification by sex

\begin{tabular}{|c|c|c|c|c|}
\hline Stratification variable & Cases, n (\%), N=68, I 59 & Controls, n (\%), N=68, I59 & OR crude $(95 \% \mathrm{Cl})$ & OR adj* $(95 \% \mathrm{CI})$ \\
\hline Male population & $53,736(100)$ & $53,736(100)$ & & \\
\hline \multicolumn{5}{|l|}{ Hypothyroidism } \\
\hline No & $52,547(97.8)$ & $52,870(98.4)$ & I (Reference) & I (Reference) \\
\hline Yes & $1,189(2.2)$ & $866(1.6)$ & $1.39(1.27-1.52)$ & $1.15(1.04-1.28)$ \\
\hline Current diagnosis & $138(0.3)$ & $81(0.2)$ & $1.72(1.31-2.26)$ & $1.33(0.98-1.81)$ \\
\hline Past diagnosis & $|, 05|(2.0)$ & $785(1.5)$ & $1.35(1.23-1.49)$ & $1.14(1.02-1.26)$ \\
\hline \multicolumn{5}{|l|}{ Hyperthyroidism } \\
\hline No & $53,4 \mid 4(99.4)$ & $53,502(99.6)$ & I (Reference) & I (Reference) \\
\hline Yes & $322(0.6)$ & $234(0.4)$ & $1.38(1.16-1.63)$ & $1.16(0.96-1.4 I)$ \\
\hline Current diagnosis & $20(0.0)$ & $15(0.0)$ & $1.33(0.68-2.60)$ & $1.13(0.53-2.38)$ \\
\hline Past diagnosis & $302(0.6)$ & $219(0.4)$ & $1.38(1.16-1.64)$ & $1.17(0.95-1.42)$ \\
\hline Female population & $14,423(100)$ & $14,423(100)$ & & \\
\hline \multicolumn{5}{|l|}{ Hypothyroidism } \\
\hline No & 12,655 (87.7) & $|3,08|(90.7)$ & I (Reference) & I (Reference) \\
\hline Yes & I,768 (I2.3) & I,342 (9.3) & $1.37(1.27-1.48)$ & $1.07(0.98-1.17)$ \\
\hline Current diagnosis & $113(0.8)$ & $83(0.6)$ & $1.41(1.06-1.87)$ & $1.28(0.92-1.79)$ \\
\hline Past diagnosis & I,655 (II.5) & I,259 (8.7) & $1.37(1.27-1.48)$ & $1.06(0.97-1.16)$ \\
\hline \multicolumn{5}{|l|}{ Hyperthyroidism } \\
\hline No & $|4,05|$ (97.4) & |4,080 (97.6) & I (Reference) & I (Reference) \\
\hline Yes & $372(2.6)$ & $343(2.4)$ & $1.09(0.94-1.26)$ & $0.98(0.82-1.17)$ \\
\hline Current diagnosis & $20(0.1)$ & $18(0.1)$ & I.II (0.59-2.II) & $0.89(0.42-1.89)$ \\
\hline Past diagnosis & $352(2.4)$ & $325(2.3)$ & $1.09(0.93-1.27)$ & $0.98(0.82-1.18)$ \\
\hline
\end{tabular}

Notes: Current diagnosis: last diagnosis within 180 days prior to (excluding) index date. *Adjusted for body mass index category, smoking status, alcohol consumption, hypertension, renal failure, congestive heart failure, and ischemic heart disease.

Abbreviations: adj, adjusted; $\mathrm{Cl}$, confidence interval; OR, odds ratio. 
Clinical Epidemiology is an international, peer-reviewed, open access, online journal focusing on disease and drug epidemiology, identification of risk factors and screening procedures to develop optimal preventative initiatives and programs. Specific topics include: diagnosis, prognosis, treatment, screening, prevention, risk factor modification,

Submit your manuscript here: https://www.dovepress.com/clinical-epidemiology-journal
Dovepress

systematic reviews, risk and safety of medical interventions, epidemiology and biostatistical methods, and evaluation of guidelines, translational medicine, health policies and economic evaluations. The manuscript management system is completely online and includes a very quick and fair peer-review system, which is all easy to use. 